\title{
Multiple organ retrieval in a brain dead left ventricular assist device donor
}

\author{
Antonio Loforte ${ }^{1}\left(\right.$ ID $\cdot$ Federica Odaldi ${ }^{2} \cdot$ Marianna Berardi $^{1} \cdot$ Silvia Boschi $^{1} \cdot$ Luciano Potena $^{3} \cdot$ Gregorio Gliozzi $^{1}$. \\ Giulio Giovanni Cavalli ${ }^{1} \cdot$ Matteo Ravaioli $^{2} \cdot$ Matteo Cescon $^{2} \cdot$ Davide Pacini $^{1}$
}

Received: 28 June 2020 / Accepted: 6 October 2021 / Published online: 15 October 2021

(c) The Japanese Society for Artificial Organs 2021

\begin{abstract}
Left ventricular assist device (LVAD) support in donors may contribute in preserving proper haemodynamics and systemic perfusion during organ retrieval thus decreasing the risk of multiple organ injury. This is an option to expand the current organ supply. We report on intra-abdominal organs procurement strategy in a selected LVAD recipient who suffered a fatal cerebrovascular accident at the time of COVID-19 pandemic outbreak. The liver and kidneys grafts have been successfully transplanted.
\end{abstract}

Keywords Heart failure $\cdot$ Brain death donation $\cdot$ Left ventricular assist device $\cdot$ Multiple organ retrieval

\section{Introduction}

Left ventricular assist device (LVAD) support in donors may contribute in preserving proper haemodynamics and systemic perfusion during organ retrieval thus decreasing the risk of multiple organ injury. This is an option to expand the current organ supply [1-5].

We report on intra-abdominal multiple organ procurement strategy in a selected LVAD recipient who suffered a fatal cerebrovascular accident. Surgery was performed at the time of COVID-19 pandemic outbreak [6, 7].

\section{Case report}

The donor patient was a brain-dead 47-year-old Afro-American woman supported by a continuous-flow LVAD system.

Antonio Loforte

antonioloforte@yahoo.it

1 Cardiac Surgery Division, S. Orsola University Hospital, IRCCS Bologna, Via Massarenti n.9, 40138 Bologna, Italy

2 General Surgery Division, S. Orsola University Hospital, IRCCS Bologna, Bologna, Italy

3 Cardiology Division, S. Orsola University Hospital, IRCCS Bologna, Bologna, Italy
The patient suffered refractory heart failure due to an end-stage dilated idiopathic cardiomyopathy, and received a HeartWare HVAD $^{\circledR}$ (HeartWare Inc., Miami, FLA; Medtronic Inc., Massachussets, MN) on April 2017, as bridge to heart transplantation (BTT). The early post-operative period had been uneventful and the patient re-joined a quite normal out-of-hospital daily life.

Since a year, the patient reported recurrent episodes of infection of driveline wound site thus requiring recurrent hospitalizations, frequent lead site dressings and intravenous antibiotic drips.

The LVAD had been running since 1048 days when a sudden patient loss of consciousness and full motor function deficit occurred due to severe haemorragic stroke as detected by computed tomography (CT) scan at the Intensive Care Unit (ICU) of a peripheral hospital to which she was emergently driven. Patient's neurological condition was irreversible and devasting as the reported Glasgow Coma Scale was 1.

The possibility of organ donation was approached by ICU physicians and the patient's family agreed to donate the patient's organs.

Our mechanical circulatory support (MCS) team, consisting of one VAD coordinator, one cardiologist, one experienced heart surgeon and one surgeon in training, joined the donor hospital to talk with donor's family, as well, check the proper LVAD working, provide a adequate monitoring 
system, and train the local physicians in how to manage the LVAD system.

After the official confirmation of brain death, despite the LVAD running, the donor was transferred from ICU to the operating theatre (OR) for abdominal multiple organ procurement setting.

The LVAD patient was systemically heparinized (10.000 UI), to avoid clotting formations in the pump. Moderate dosage of vasoactive drugs infusion (norepinephrine $0.18 \mathrm{mcg}$ / $\mathrm{Kg} / \mathrm{min}$ ) and positive fluid balance were necessary to guarantee a proper LVAD filling and functioning.

The laboratory values $6 \mathrm{~h}$ before procurement were: serum sodium $145 \mathrm{meq} / \mathrm{L}$, serum creatinine ( $\mathrm{SCr}$ ) $0.97 \mathrm{mg} /$ $\mathrm{dL}$, total bilirubin $2.1 \mathrm{mg} / \mathrm{dL}$, serum alanine aminotransferase (ALT) $25 \mathrm{IU} / \mathrm{L}$, aspartate aminotransferase (AST) $12 \mathrm{IU} / \mathrm{L}$ and international normalized ratio (INR) 2.5. Viral serologies and blood cultures were negative at the time of organ procurement and afterwards.

LVAD donor organ retrieval happened at the same day of the virus SARS-Cov-2 designation as a pandemic by the World Health Organization (WHO) [6, 7].

Real-time reverse transcription polymerase chain reaction (RT-PCR) test of 2019-nCoV on serum, bronchoalveolar lavage (BAL) and lower respiratory tract swab samples were performed in the donor but resulted to be negative. Arterial blood gas analysis was not hypoxemic. C-reactive protein (CPR) was $1.5 \mathrm{mg} / \mathrm{dL}$. Official authorizations by the Italian national centre for transplantation (CNT) and Ministry of Health have been met. No contra-indications for SARS-CoV-2-negative donors have been evident in transplant surgery [7]. All potential transplant recipients were informed.

Liver and kidney surgeons joined the operation together with two heart surgeons [1]. The patient had a previous sternotomy and the LVAD was fully intra-thoracic. A xyphoid to pubis midline incision and a full laparotomy were performed by paying attention to preserving the proximal tunnelling tract of the LVAD driveline $[1,2]$ which was directed distally towards the right upper abdomen just below the muscles fascia. All donor surgery was performed whithout stopping the LVAD system, to avoid donor haemodynamic instability or cardiac arrhythmias which may be facilitated by the eventual blood back-flow from ascending aorta to left ventricle through the LVAD outflow.

At the macroscopical evaluation, the liver had a normal consistency, with sharp margins and a smooth surface.

Systemic heparinization was reinforced (5.000 UI) to get an activated clotting time of $>300 \mathrm{~s}$. The abdominal suprarenal aorta was then cross-clamped and the LVAD was stopped $[1,8]$. Thus, the infrarenal distal aorta was directly cannulated and crystalloid cold Servator C (S.A.L.F. S.p.a., Bergamo, Italy) solution was infused through the aortic cannula $[1-3,8]$. Transection of the caval vein near its abdominal bifurcation was performed to vent the cold solution and sterile ice was applied in the abdominal cavity.

As required by the hepatic surgical team, the heart surgeons performed the re-sternotomy and the mediastinal adesions debridment to better expose all abdominal organs thus allowing a larger graft recruitment of the supra-diaphragmatic caval vein. The hepatectomy and bilateral nepherectomy were accomplished by the abdominal surgeons, traditionally $[1-3,8]$.

Time from support withdrawal to total extraction time (hepatectomy and nephrectomy) was 42 min (cold ischaemic time). Seven litres of Servator C solution were infused through the aortic cannula and $2 \mathrm{~L}$ were also infused through the portal vein, celiac axis and renal arteries for flushing [1-3].

The liver was transplanted in a tiny 63-year-old female who had undergone a distal pancreatectomy due to an aggressive neuroendocrine tumour which involved the liver after surgery, secondarily.

The left kidney was transplanted in a 47-year-old man with dialysis-dependent end-stage renal disease with a previous non-functioning kidney transplant. The right kidney was transplanted in a 59-years-old woman with dialysis- dependent end-stage renal disease due to diabetic nephropathy.

No COVID-19 disease [6, 7] occurred in the transplanted patients. Real-time RT-PCR of 2019-nCoV on recipients serum resulted to be negative, thereafter. The biopsies which have been performed in the transplanted grafts excluded any typical COVID-19 related vasculitis and/or diffuse endothelial damage $[6,7]$.

\section{Discussion}

The number of reports describing organ retrieval from donors with LVADs is still small [1-5].

Despite the current adoption of newer continuous-flow pumps, LVAD recipients are still exposed to risks of infectious and neurological adverse events [8]. As such patients appreciate the need for donor organs at the time of their LVAD implantation, they themselves are usually willing to be donors if they themselves become brain dead $[1,4]$.

The suitability of patients with LVADs for organ donation has not been addressed in terms of guidelines [1, 4]. Considering prior cardiac failure and cardiac surgery, it is a relative contraindication [1-5]. However, after LVAD implantation, all systemic organs could be kept stable. Thus, if organ damage from severe heart failure can be avoided, patients with an LVAD can be organ donors even if considered marginal [1-5].

Technically, sternal re-entry, exposure of the device and organs in the thoracic cavity may be rescue expecially if lung grafts are needed $[4,5]$. In this case, the lungs were 
not addressed due to pulmonary oedema and COVID-19 outbreak. The donor hospital was $10 \mathrm{~km}$ far from the referral MCS centre and two dedicated heart surgeons had the chance to join the OR. They knew how the LVAD had been implanted and where the driveline and outflow graft were because they had implanted the LVAD for this donor, and they performed the dissection and exposure of the device [1-3].

LVAD support may reduce the risk of unpredictable cardiac arrest and arrythmias [1-4]. However a adequate LVAD system knowledge and a specific haemodynamic managenent may be required. In this case the referral MCS team consisting of a cardiologist, a VAD coordinator and two heart surgeons joined the donor hospital for assistance.

Kidneys and livers procured from brain death donors on ECMO perform similarly to non-ECMO organs even though livers from ECMO donors have a higher discard rate than non-ECMO donors [2, 3]. Selected LVAD donors represent an alternative option to increase the chance of transplantation [1-5]. By adoption of the above technique, any warm ischaemic time is avoided as the LVAD is stopped at the time abdominal aorta cross-clamping.

COVID-19 disease was excluded by PCR tests on donor BAL and serum. None of transplanted patients received 2019-nCoV antiretroviral therapy in the post-operative period [6,7]. No specific triage setting has been adopted [7] and all patients gave consensus on this.

\section{Conclusions}

Our report confirms that successful retrieval of intra-abdominal organs can be successfully achieved in selected LVAD donors. A multidisciplinary team which involves a dedicated MCS team contributes in providing a adequate LVAD donor management before and during multiple organ retrievial.

\section{Declarations}

Conflict of interest The paper is not under consideration elsewhere, none of the paper's contents have been previously published, and all authors have no conflict of interest to declare. The manuscript has been read and approved by all the authors; requirements for authorship have been met. Each author believes that the manuscript represents an honest work.

\section{References}

1. Rayhill SC, Martinez-Mier G, Katz DA, Kanchustambam SR, Wu YM. Successful non-heart-beating donor organ retrieval in a patient with a left ventricular assist device. Am J Transpl. 2004:4:144-6.

2. Munawar K, Rajagopalan N, Grigorian A, Dennis D, Guglin M. Successful liver transplantation from a donor with a continuousflow left ventricular assist device for 9 months. Transpl Proc. 2020;49:2406-8.

3. Carter T, Bodzin AS, Hirose H, et al. Outcome of organs procured from donors on extracorporeal membrane oxygenation support: an analysis of kidney and liver allograft data. Clin Transpl. 2014;28:816-20.

4. Matsumoto K, Yamasaki N, Tsuchiya T, et al. Single lung retrieval from a donor supported by a left ventricular assist device. J Thorac Dis. 2017;9:E685-8.

5. Schiavon M, Calabrese F, Di Gregorio G, et al. Ex-vivo recruitment and $\mathrm{x}$-ray assessment of donor lungs in a challenging retrieval from a donor supported by LVAD using the portable normothermic perfusion system: a case report. J Cardiothorac Surg. 2017;12:30.

6. WHO: Rolling Updates on Coronavirus Disease (COVID-19). https://www.who.int/emergencies/diseases/novel-coronavirus2019/events-as-they-happen. Accessed 30 Oct 2020.

7. Shah MB, Lynch RJ, El-Haddad H, Doby B, Brockmeier D, Goldberg DS. Utilization of deceased donors during a pandemic: argument against using SARS-CoV-2-positive donors. Am J Transpl. 2020;20:1795-9.

8. Starzl TE, Miller C, Broznick B, Makowka L. An improved technique for multiple organ harvesting. Surg Gyn Obstet. 1987; 165:343-8.

Publisher's Note Springer Nature remains neutral with regard to jurisdictional claims in published maps and institutional affiliations. 